\title{
3D Laser Range Scanner with Hemispherical Field of View for Robot Navigation
}

\author{
Julian Ryde and Huosheng $\mathrm{Hu}$
}

\begin{abstract}
For mobile robots to be of value in practical situations a 3D perception and mapping capability will almost always prove essential. In this paper a 2D laser scanner is modified to produce 3D scans with a resolution of one degree updated every 3 seconds. This result is achieved by adding a rotating mirror to the original scanner in an inexpensive and relatively simple modification that is easily made to a standard mobile robot. The modified robot is then able to produces 3D scans at a various frequencies up to $1 \mathrm{~Hz}$ and accurate to $0.02 \mathrm{~m}$ over an $8 \mathrm{~m}$ range.
\end{abstract}

Index Terms-3D laser scanner, mobile robots, range sensors

\section{INTRODUCTION}

It has proved difficult to provide a robot with the means of obtaining reliable, 3D information about its environment. So far the two main approaches have been based upon vision and/or laser range finding. Both have been dogged by problems. Vision is often computationally intensive and suffers from sensitivity to changes in illumination. Another source of difficulty stems from the need to solve the correspondence problem and although this can be reduced to some extent by adopting structured light approaches, data spatial density does not approach that delivered by laser scanners. So far nonvisual localisation and mapping has taken place in $2 \mathrm{D}$. The main reason for this in the case of laser range finders is the limitations imposed by the sensors. In the case of vision the 2D limitation has been dictated by limitations in processor speed and the resource intensive nature of the algorithms needed for stereoscopic vision.

Recently in an effort to tap the advantages of 3D sensing, researchers have mounted 2D laser scanners on nodding or rotating mechanisms [1][2][3][4]. What all of these implementations have in common is that they necessitate in various ways the motion of the entire 2D scanner. Whilst such innovations have tended to deliver good quality data they have done so at the cost of significant hardware complexity and concomitant mass and power consumption increases. A popular, alternative approach has seen two laser scanners mounted with their scan planes orthogonal to one another [5]. However whilst it must be accepted that the orthogonal mounting technique can produce 3D maps it does not give the robot true 3D sensory perception and this is essential for reliable obstacle avoidance and local path planning. Significant attention is now being focused on 3D laser mapping [6][7][8].

Julian Ryde is with the Autonomous Systems Laboratory, CSIRO ICT Centre, PO Box 883, Kenmore, QLD 4069, Australia. Huosheng Hu is with the Department of Computing and Electronic Systems, University of Essex, Wivenhoe Park, Colchester CO4 3SQ, U.K. (E-mail: julian.ryde@csiro.au, hhu@essex.ac.uk). This work was supported by the EPSRC.
There are numerous commercial 3D laser range finders in production but these are invariably expensive an appreciable factor when considering applications involving several robots. In addition commercial range finders are usually aimed at the needs of surveyors and tend to have a narrow field of view sacrificing scan rate for high point density. The very fact of the number and variety of approaches is a clear illustration of the rapidly increasing interest in developing full, 3D sensory perception for mobile robots in particular.

The benefits of full 3D mapping are manifold and so the rapid expansion of this field is inexorable. The detection of negative and over-hanging obstacles greatly improves avoidance behaviour. Once 3D maps of an environment have been built they can be customised for needs of, and distributed to, different robots. For instance various 2D occupancy grids may be created for robots of different sizes or which have 2D sensors at different heights. Severely cluttered and chaotic environments such as those attending search and rescue operations may be mapped reliably. Maps that have been based upon the ceilings of rooms [9] will remain accurate for longer and an unobstructed view of the ceiling is often easily accessible to a robot even in cluttered environments [10]. A different approach that takes advantage of full 3D perception [11] uses virtual $2 \mathrm{D}$ scans produced by projecting the $3 \mathrm{D}$ data vertically into a plane and then taking the furthest point for each 2D scan angle. Although this technique produces good 2D representations in cluttered environments is suffers from the usual disadvantages of 3D sensing technologies - viz slower scan acquisition time and a geometric increase in the amount of data needing to be processed. Some approaches combine both vision and a 3D laser range finder to generate fully textured 3D models of the environment [12].

\section{3D SCANNER DESIGN}

\section{A. Hardware Design}

Instead of nodding or rotating the laser scanner which introduces difficulties in hardware implementation and high power consumption, we adopted the rotating mirror mechanism to produce $3 \mathrm{D}$ scans with a field of view up to $120^{\circ}$ by $270^{\circ}$ (2), as shown in Fig. 1. It is light, has low power consumption and is easily deployed on conventional robotic platforms. Its accuracy of the laser scanner is fundamentally dependent on precise knowledge of the mirror position. There are two main methods to achieve this rotational accuracy: (i) The closed feedback control loop method that requires a angular sensor to measure the angular velocity of the motor to maintain a constant mirror angle velocity and in turn 

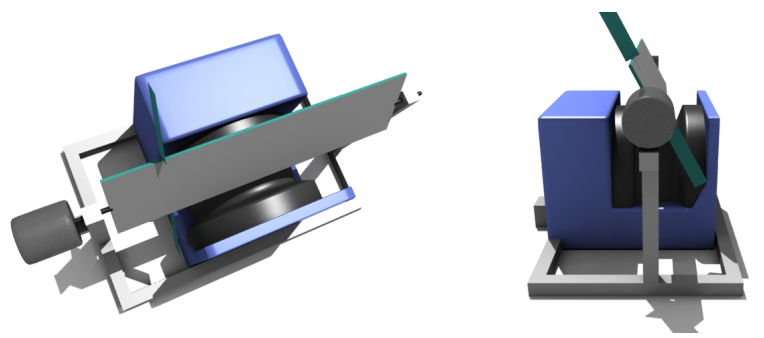

Fig. 1. Top and side views of the enhanced 3D laser scanner. The blocking arms affixed to the mirror edge provide the angular position of the mirror through the laser data.

accurate mirror position; (ii) The open loop control method that requires reliable and consistent driving mechanism over mirror revolutions. We have chosen the open loop control in this research for simplicity. It is accomplished to high accuracy with a quartz oscillator driving a high resolution stepper motor. The closed loop scanner is accomplished with a standard DC motor and a rotation sensor.

The use of a mirror has a number of advantages, namely less power consumption and simpler hardware. The disadvantages are a reduced scan angle to around $120^{\circ}$, a blind spot when the mirror is edge on and a requirement for more complex geometric calculations (Fig. 2) to correct for the separation between the mirror and the scanner. In Fig. 2 the effect of the mirror is to bend part of the $x y$-coordinate plane to a new elevation.

\section{B. Data Processing Software}

The SICK LMS 200 laser scanner is configured to scan a $180^{\circ}$ arc at $1^{\circ}$ intervals. These settings were chosen to minimize the number of scan points in order to increase the complete scan frequency. The scan frequency is limited by the serial data rate to $0.05 \mathrm{~Hz}$. This data rate is $34.8 \mathrm{~kb} / \mathrm{s}$, the maximum for the serial interface, resulting in 13 horizontal scans per second. The SICK LMS 200 can support a $500 \mathrm{~kb} / \mathrm{s}$ data rate using a USB interface. At this data rate, full 3D $1^{\circ}$ by $1^{\circ}$ scans are possible at $0.5 \mathrm{~Hz}$ (Section II-C).

The mirror is displaced forward from the origin of the laser scanner. This displacement alters the range readings by a value dependent on the angle of the mirror. The following equations, which reference the values indicated in Fig. 2, indicate the conversion between the $r, \theta$ and $\phi$ coordinates, measured by the laser scanner, and 3D Cartesian coordinates.

$$
\mathbf{x}=\left(\begin{array}{c}
(r \cos \theta-d) \cos \phi+d \\
r \sin \theta \\
(r \cos \theta-d) \sin \phi
\end{array}\right)
$$

where the value $d$ is the separation between the origin of the laser scanner and the axis of the rotating mirror. The range and bearing as measured by the laser scanner are $r$ and $\theta$. The angle of the plane to the horizontal introduced by reflecting the laser ray from the rotating mirror in front of the scanner is indicated by $\phi$. The frequency of angular position reports can be adjusted by altering the number of teeth on the blocking wheel and by changing the gearing. A high angular position update rate leads to better accuracy in that the system can

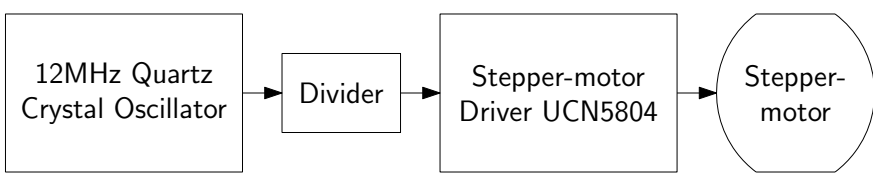

Fig. 3. Block diagram illustrating the electronic components of the 3D laser scanner.

respond faster to changes in angular velocity. However, missed counts do still occur occasionally.

\section{Enhanced 3D Laser Scanner}

The laser scanner described above can be deployed to standard mobile robots without any hardware modifications. Although it is very convenient, some relatively minor modifications to the hardware of the standard mobile robotic platform bring about substantial improvements. The main features of the proposed design are:

- Fig. 1 shows the enhanced 3D laser scanner is based on a SICK LMS 200 [13] [14] [15] which is facing upwards. A rotating mirror driven by a stepper motor via a signal from a quartz crystal oscillator shown in Fig. 3.

- The high update rate $(75 \mathrm{~Hz})$ of the LMS 200 means the 3D scanner delivers scans at $1 \mathrm{~Hz}$ with a horizontal resolution of $1^{\circ}$ and vertical resolution of $5^{\circ}$.

- Accurate determination of mirror angle is paramount to the accuracy of the 3D laser scanner.

- Finally the most difficult problem to address is that DC motors used in this research have significant amounts of backlash.

A better approach which solves these problems is to drive the mirror using a stepper motor directly coupled to the mirror spindle. Stepper motors can be operated over a range of speeds and are especially suited to low speed operation. The stepper motor used in this research has a $1.8^{\circ}$ angular resolution that is half-stepped to give 400 steps per revolution. Although the stepper motor, when driven by a quartz crystal oscillator signal, has an exceedingly consistent angular velocity the mirror still needs to be approximately balanced. Significant off-centre mass distribution will cause flexing to occur between the motor connection and the mirror. This flexing causes significant distortion in the scan data which may be reduced by using stiffer materials and balancing the mirror to reduce the variation in load torque. Generally, the stepper motor mechanism is much less sensitive to variations in load torque than the DC motor driven system.

A block diagram of the electronic components in Fig. 3 illustrates how a $12 \mathrm{MHz}$ oscillator signal is stepped down to produce a highly stable and accurate $15 \mathrm{~ms}$ clock signal. The clock signal drives the stepper-motor driver microchip which outputs the correct waveform for the stepper-motor. This configuration coupled with an adequately balanced rotating mirror mechanism results in a very consistent rotation speed over an extended period of time.

The field of view is improved by placing the laser scanner facing upwards, Fig. 1. The blocking arms affixed to the mirror edges in Fig. 1 and Fig. 5 alter the range reading of 


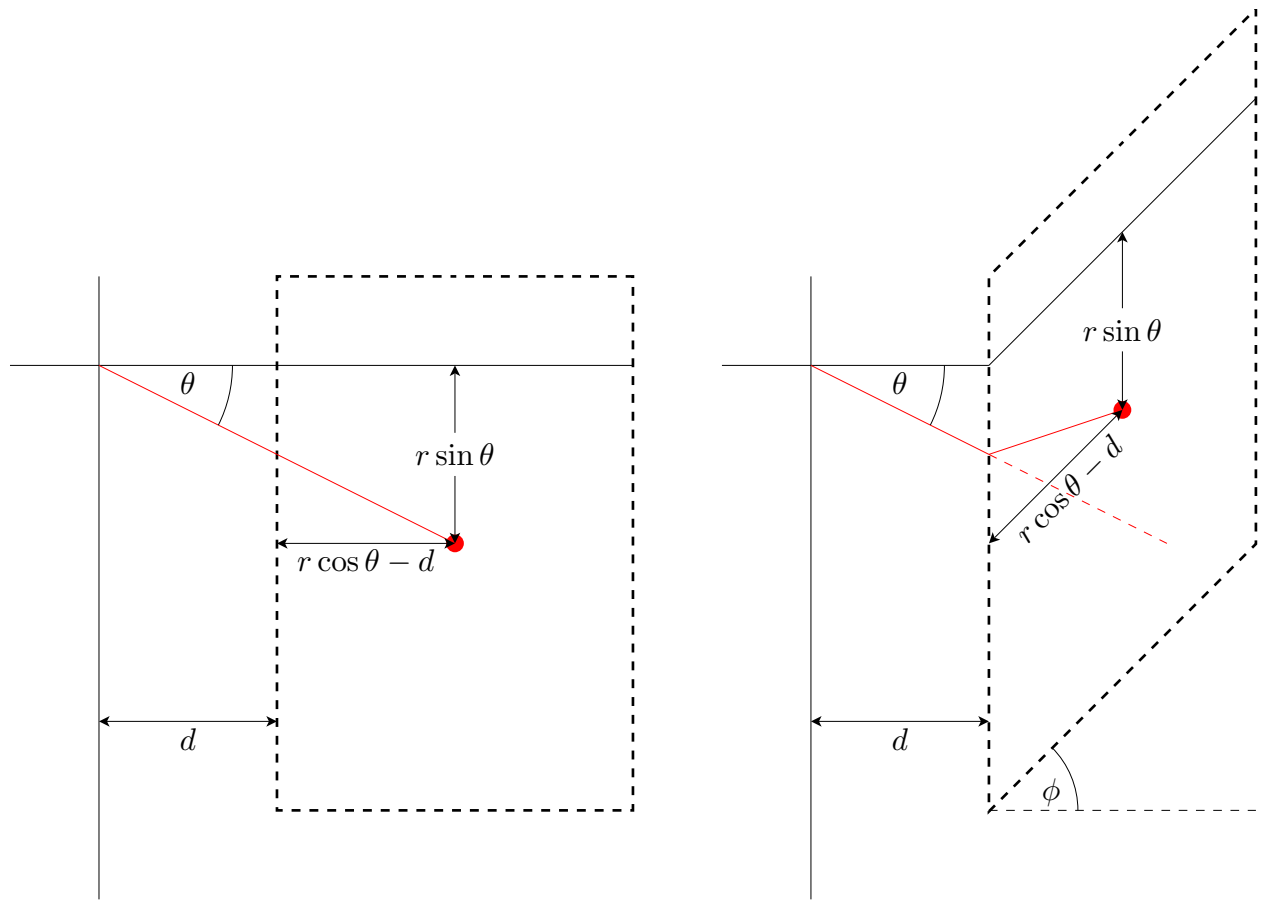

Fig. 2. Calculating 3D coordinates from range data. The plane is elevated by an angle $\phi$ to the page. The line at $\theta$ to the $x$-axis shows the laser as it is deflected into the plane by the mirror. The range reading returned by the scanner is $r$ and angle $\theta$.

the rightmost laser ray twice per mirror revolution and once per scan. This allows the pitch of the scan to be corrected and has the added advantages of simplifying the design by embedding the feedback of rotational position in the laser scans themselves thus ensuring the that angular position is synchronised with the correct laser data. Thus there is no need for an additional data connection to the host robot meaning that existing systems maybe augmented with the rotating mirror very easily as all the information required to construct the $3 \mathrm{D}$ scans is in present in the laser range readings.

In this way the blind spot when the mirror is edge on is rotated to point upwards rather than in front of the robot. Most mobile robots need to see in front rather than above. The side blind lobes are unaltered and the observable volume is given by the following constraints.

$$
0.5<r<8,25<\theta<155,75<\phi<105,
$$

Data from a single scan of a small room is plotted in Fig. 6. The room is a cuboid and with flat ceiling, walls and floor. The scanner records ranges from almost all directions around the robot. The quality of the data returned can be visually verified by inspection of the straight edges of the room in Fig. 6.

The SICK LMS 200 is capable of operating at higher data rates than can be delivered by a standard serial link. The internal mirror rotates at $75 \mathrm{~Hz}$ and this is the natural data frequency if the RS232 serial communications bottleneck is removed. The SICK LMS supports a high-speed serial connection RS422 which may be connected to a standard USB port with a USB-RS422 converter. This allows $180^{\circ}$ of range data at $1^{\circ}$ resolution to be delivered to the host computer at $75 \mathrm{~Hz}$. The horizontal resolution is fixed at $1^{\circ}$ however the vertical resolution may be adjusted by varying the speed of the external mirror rotation. Matching the vertical resolution to the horizontal resolution gives scan times of $360 / 75=4.8$ seconds. The resulting scans are high detail and can be used for map building, however for real-time obstacle avoidance a faster mirror angular velocity is more suitable and scan frequencies of $1 \mathrm{~Hz}$ are feasible.

\section{3D SENSOR MOdEL}

A sensor model is required to make predictions about what data would be returned by the scanner at various poses within a map. The 3D scanner returns a list of coordinates corresponding to all the range returns detected. The number of elements in this list varies because laser measurements may not be returned at all for some angles. An approach similar to [16] where the voxels traversed by a vector is considered. This is often referred to as the ray trace sensor model and is particularly suited to laser range data even though [16] employ it for stereo vision range data, due to the collimated nature of the laser and because the beam width, Fig. 4, is less than the map resolution. This process is summarised in Algorithm 1.

\section{A. Ray Trace Sensor Model}

There are a number of deficiencies with this simplified algorithm but its persistence in mobile robots is due to its simplicity of implementation and low computational requirement. The premise is that the occupancy of the terminating voxel is increased and the occupancies of the voxels on the line between the origin of the laser ray and the terminating voxel are decreased. This process is summarised in Algorithm 1. 


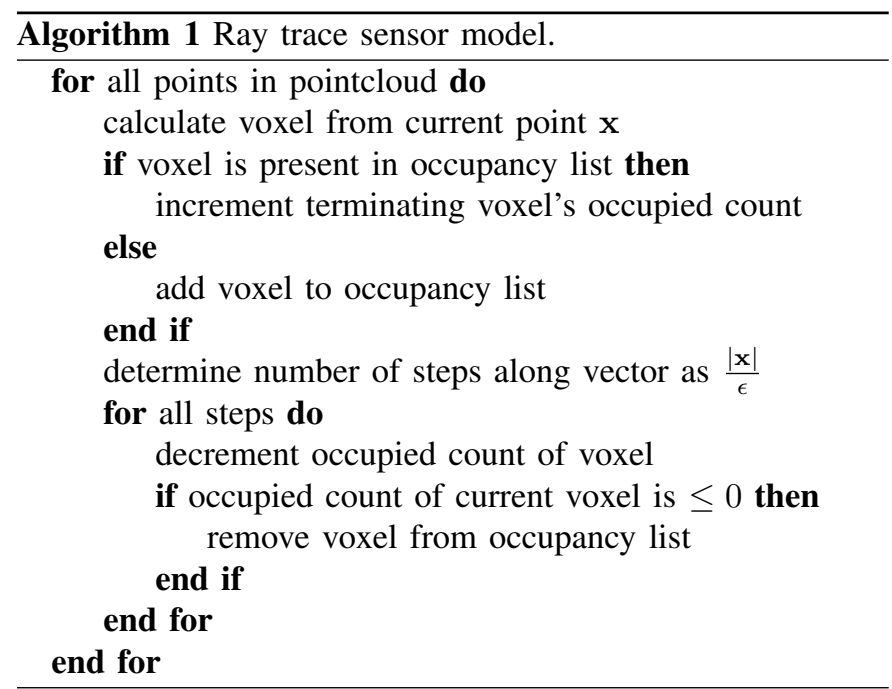

In this way, given a 3D map, pose and sensor model the expected occupancy of voxels surrounding the pose can be calculated. The accuracy of the sensor model is dependent upon the errors in the data returned by the $3 \mathrm{D}$ laser scanner. The errors of the $3 \mathrm{D}$ laser range finder may be modelled as Gaussian in $\theta, \phi$ and $r$. Complete differentiation of (1) produces the following expressions for the errors in $x, y$ and $z$.

$$
\begin{aligned}
\Delta x= & \cos \phi \cos \theta \Delta r-r \cos \phi \sin \theta \Delta \theta \\
& -\sin \phi(r \cos \theta-d) \Delta \phi+(1-\cos \phi) \Delta d \\
\Delta y= & \Delta r \sin \theta+r \cos \theta \Delta \theta \\
\Delta z= & \cos \theta \sin \phi \Delta r-r \sin \theta \sin \phi \Delta \theta \\
& -\sin \phi \Delta d+r \cos \theta \cos \phi \Delta \phi-d \cos \phi \Delta \phi
\end{aligned}
$$

Converting from the spherical polar coordinate to the Cartesian coordinate system produces errors that are linearly dependent on the distance from the origin. This is superior to the quadratic dependence found in triangulation techniques. This spread in uncertainty is useful for inclusion in the sensor model. The unmodified $2 \mathrm{D}$ scanner is capable of reading ranges up to $80 \mathrm{~m}$. Such large distances are infrequently observed in indoor environments however and as the error scales linearly with range the accuracy of these long range readings is poor. To ease the complexity of the sensor model the readings are truncated to $8 \mathrm{~m}$ and the upper bound of the error, namely that at $8 \mathrm{~m}$, is assumed for all remaining range readings. This error is then intrinsically incorporated into the sensor model by limiting the map resolution to this value.

\section{B. Pixel Mixing}

Pixel mixing is systematic error that arises from the width of the laser beam. The laser scanner operates at a wavelength of $880 \mathrm{~nm}$ in the infrared part of the electromagnetic spectrum and is consequently invisible to the human eye. Fig. 4 contains images of the laser scanner taken with an infrared sensitive camera. In order to emphasise the laser beam, two images of the scanner, off and operating, are shown for comparison. The diameter of the infrared beam is around $0.01 \mathrm{~m}$ as seen
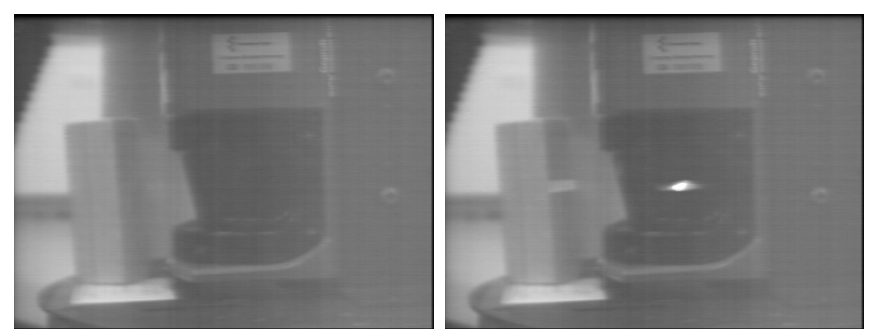

Fig. 4. Infrared image of the SICK LMS 200 laser scanner in operation showing the width and position of the laser beam.

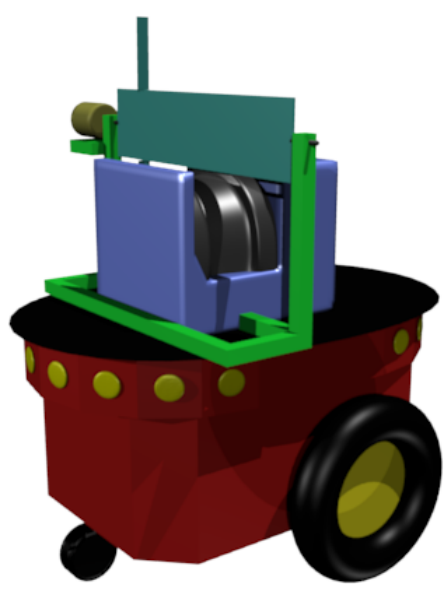

Fig. 5. Pioneer II robot with rotating mirror mechanisms enabling 3D scans.

in Fig. 4 where the beam is incident upon the wooden block. Fig. 4 indicates the height at which the centre of the rotating mirror needs to be placed. The beam width places a limit on the resolution of the map and plays a role in the selection of the ray trace sensor model. Due to the $0.01 \mathrm{~m}$ beam diameter it is clearly not worth mapping to a better resolution. Indeed, experimental data supports this with range errors of the order of $0.02 \mathrm{~m}$ under standard conditions, even though there is a $\mathrm{mm}$ range resolution setting.

In practice however these errors may persist for some time before they are removed. It is more efficient to remove these errors in the preliminary stages of data acquisition. In 3D occupancy grids pixel mixing tends to produce isolated occupied cells where there are discontinuities in the range readings with respect to $\theta$ and $\phi$. Successful filtering to remove pixel mixing relies on the detection of these range discontinuities and corresponding isolated voxels.

For the 3D scanner pixel mixing problems are more acute for vertical range discontinuities. The laser scanner is designed to accommodate and correct such horizontal errors because that is the direction the laser beam is rotated by the 2D laser scanner. The vertical motion arising from a external mechanism was not anticipated by the manufactures and consequently no error correction is applied vertically by the internal electronics.

\section{EXPERIMENTAL RESUlts AND ANALYSis}

The Pioneer II mobile robot was used in the experiments, and is equipped with the enhanced 3D laser scanner. The errors 
in data produced by the 3D scanner are measured in a number of manners. An estimate of the errors is generated by scanning a room with ceiling, walls and floor. Systematic errors are clearly distinguished as curvature in what should be planar surfaces. Once the points are coplanar then the random error is assessed in the following manner.

A subset of the data points associated with the ceiling which is $3.2 \mathrm{~m}$ above the origin of the $3 \mathrm{D}$ laser scanner is extracted from the scan. The standard deviation of the $z$ coordinates for this subset gives a good estimate of the error in $z$ at this height. Clearly, the error may vary with $z$ and a more thorough method is explained later. This extract indicates that the error in $z$ is approximately $0.02 \mathrm{~m}$. Repeating this analysis for the smooth walls of the room indicate a similar error in $x$ and $y$. Repeated measurements at different positions reveal that the error is relatively independent of distance over the range $0-8 \mathrm{~m}$ implying that a simplified sensor model with constant error is appropriate. In this simplified sensor model the errors are $0.02 \mathrm{~m}$ regardless of distance. This also places a lower bound on the map resolution.

To establish the size of the systematic error numerous dimensions of the experimental environment are compared to the generated map and the mean error is $0.07 \mathrm{~m}$ on dimensions of $6 \mathrm{~m}$. The systematic error results would seem to indicate that there is no point using map resolutions better than $0.07 \mathrm{~m}$ and experimentally we find the best localisation and mapping performance with resolutions of $0.08 \mathrm{~m}$. It is worth noting that local regions of the scans are accurate to $0.02 \mathrm{~m}$. For the enhanced laser scanner driven by a stepper motor the systematic error drops to $0.05 \mathrm{~m}$. Example data returned from the enhanced 3D scanner is rendered in Fig. 6.

A more thorough estimate of the random error is available by taking repeated scans at the same pose within static surroundings. Ideally the scans should be identical because nothing in the environment has changed between the scan times. The differences between the two scans are due to random error as any systematic distortions will be equally present in both scans. For each point in the first scan the distance to the closest point in the second scan is recorded.

The distribution of these corresponding closest point distances is graphed in Fig. 7. Although the mean closest point separation in Fig. 7 is $0.011 \mathrm{~m}$ the median is $0.006 \mathrm{~m}$ and the mode is $0.004 \mathrm{~m}$. The random error is remarkably small with very few separations venturing above $0.02 \mathrm{~m}$. Thus it may be concluded that the majority of the error comes from systematic errors or distortions within the laser scan. In this case flexing between the stepper motor axle and the mirror. For mobile robots the data accuracy is significantly better than that acquired by stereoscopic vision and comparable to those approaches that rotate or nod the entire 2D scanner. For medium sized robots in standard human environments this $0.02 \mathrm{~m}$ accuracy is adequate and paves the way for equally accurate mapping and localisation.

Fig. 8 juxtaposes a photograph and scan both of which are taken from the same point of view. The scan has been rendered with lighting to aid visual interpretation. From this Fig. 8 it is possible to appreciate the fidelity of the laser scanner to a true environment, as various objects apparent in the photograph

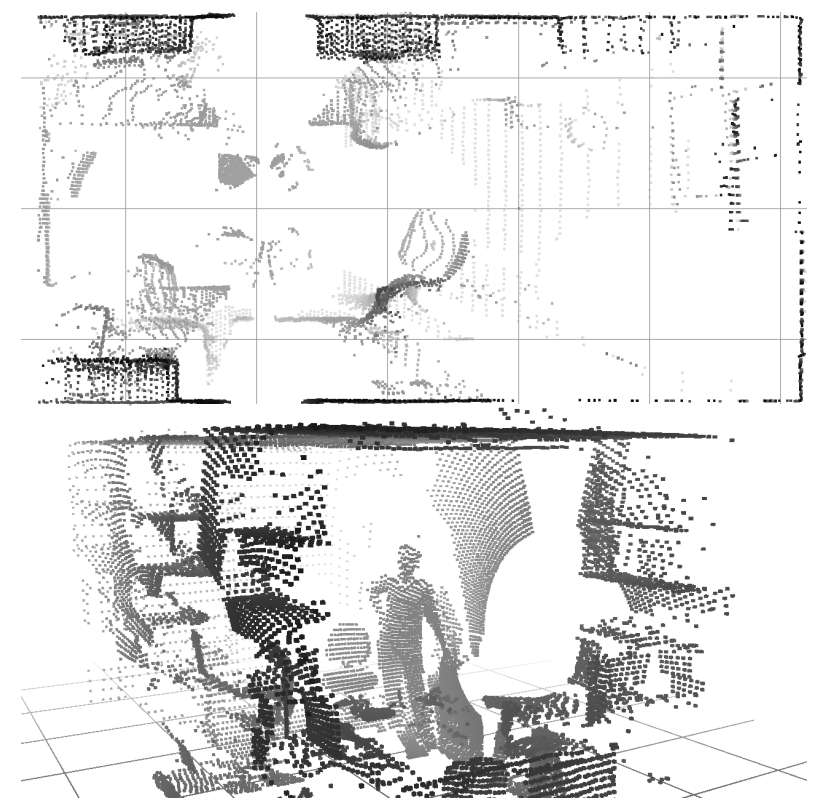

Fig. 6. Top and side views of a single scan produced by the enhanced laser scanner for a room of dimensions 3 by 6 by $2.75 \mathrm{~m}$ at $0.02 \mathrm{~m}$ resolution. The points are coloured by distance from the point of view and the grid cells are $1 \mathrm{~m}$.

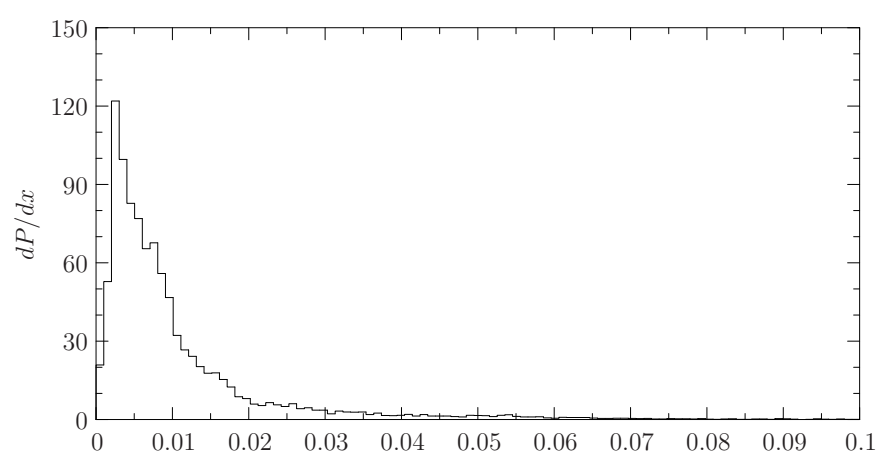

Fig. 7. Distribution of the distances(m) to the corresponding closest points in two sequential scans.

are alse clearly evident in the 3D scan. An example of such is shown in Fig. 9 displaying the resulting 3D occupancy list map produced by scan matching 40 scans generated by the enhanced 3D scanner described herein.

\section{Conclusions And Future Work}

This paper describes the enhancements made to a standard SICK LMS 200 laser scanner to produce a full 3D sensor with low power consumption (20W), minimal hardware requirements and that may be easily deployed on standard Pioneer II and other robotic platforms with little or no modification. Having shown that this approach is viable the 3D scanner is improved in a number of manners: (i) Using a USB-RS422 converter to increase the data rate from the $2 \mathrm{D}$ laser scanner to $75 \mathrm{~Hz}$; (ii) orientating the $2 \mathrm{D}$ scanner to point up into the mirror delivers a wider field of view with the blind spots placed above and to the sides of the robot, rather than in front of the robot; (iii) Driving a mirror in an open control loop manner with a stepper motor clocked by a quartz crystal oscillator 

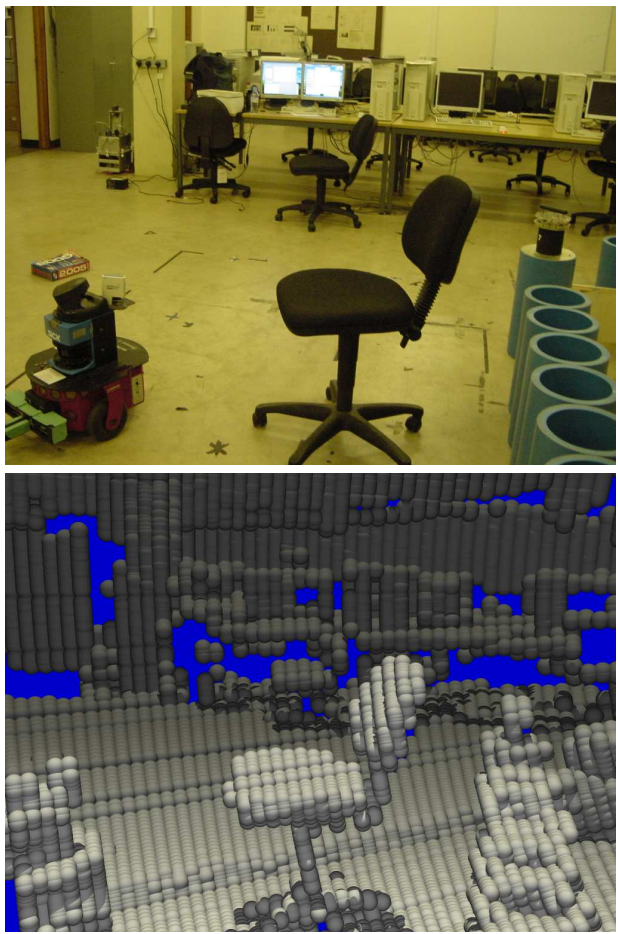

Fig. 8. Photograph and 3D scan of mapped room from the same point of view. Blue regions correspond to areas with no returned scan.

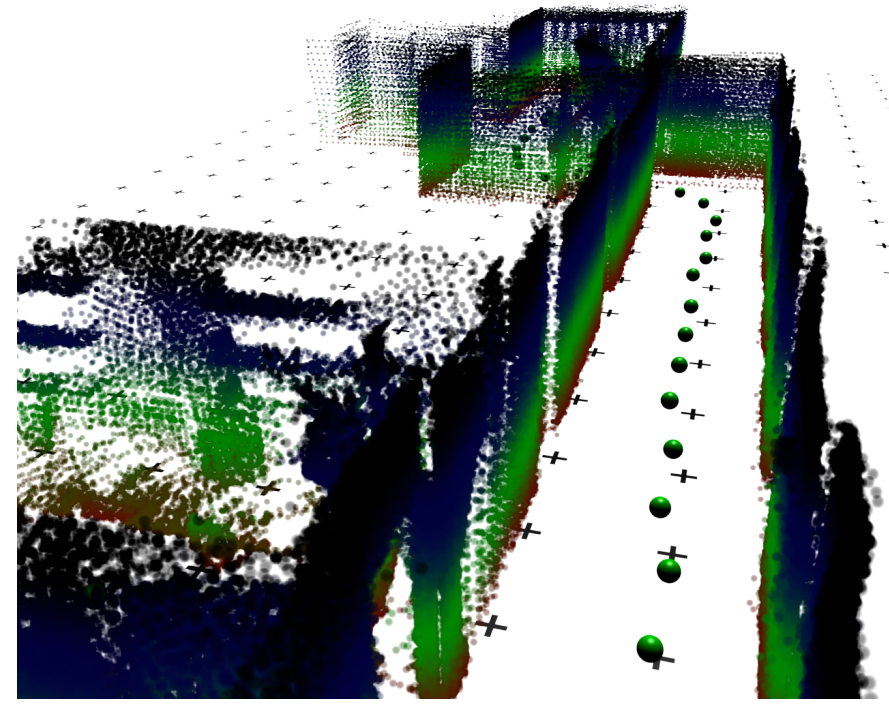

Fig. 9. Corridor view demonstrating a sequence of scan matched 3D scans. The green spheres are the observation positions and the map voxels are coloured by height.

clock signal, which has a negligible impact on the accuracy of the 2D laser scanner over the 0 to $8 \mathrm{~m}$ range resulting in an error of $0.02 \mathrm{~m}$. In this way almost omni-directional 3D scans consisting of up to 5000 data points are delivered to the robot's computer at a configurable $1 \mathrm{~Hz}$.

Our further research will deal with the representation and processing of this data in a localisation and mapping context. Repeated scanning from a fixed position may be used to determine the short term dynamic profile of an environment. If there are people moving, these will be detected as differences between successive scans and removed. An intensive field testing of the developed 3D laser scanner will be carried out in both indoor and outdoor environments.

\section{REFERENCES}

[1] K. Lingemann, H. Surmann, A. Nüchter, and J. Hertzberg, "Indoor and outdoor localization for fast mobile robots," in Proc. of the IEEE/RSJ Int. Conf. on Intelligent Robots and Systems, Sendai, Japan, Sep. 2004, pp. 2185-2190.

[2] A. Nüchter, K. Lingemann, J. Hertzberg, and H. Surmann, "Heuristic-based laser scan matching for outdoor 6D SLAM," in Advances in Artificial Intelligence. 28th annual German Conf. on AI, Sep. 2005.

[3] D. Cole and P. Newman, "Using laser range data for 3D slam in outdoor environments," in Robotics and Automation, 2006. ICRA 2006. Proceedings 2006 IEEE International Conference on, May 15-19, 2006, pp. 1556-1563.

[4] P. Pfaff, R. Triebel, and W. Burgard, "An efficient extension to elevation maps for outdoor terrain mapping and loop closing," The Int. Journal of Robotics Research, vol. 26, no. 2, pp. $217-$ 230, 2007.

[5] A. Howard, D. F. Wolf, and G. S. Sukhatme, "Towards 3D mapping in large urban environments," in IEEE/RSJ Int. Conf. on Intelligent Robots and Systems, Sendai, Japan, Sep. 2004, pp. 419-424.

[6] M. Montemerlo and S. Thrun, "Large-scale robotic 3D mapping of urban structures," in Proc. of the Int. Symposium on Experimental Robotics (ISER), 2004.

[7] A. Howard, A. Parker, E. Lynne, and G. S. Sukhatme, "The SDR experience: Experiments with a large-scale heterogenous mobile robot team (extended abstract)," Int. Symposium on Experimental Robotics, Jun. 2004.

[8] D. Hähnel, D. Schulz, and W. Burgard, "Map building with mobile robots in populated environments," in Proc. of the IEEE/RSJ Int. Conf. on Intelligent Robots and Systems (IROS), 2002.

[9] W. Jeong and K. Lee, "CV-SLAM: A new ceiling vision-based SLAM technique," in IEEE/RSJ Int. Conf. on Intelligent Robots and Systems, 2005.

[10] W. Burgard, A. B. Cremers, D. Fox, D. Hähnel, G. Lakemeyer, D. Schulz, W. Steiner, and S. Thrun, "Experiences with an interactive museum tour-guide robot," Artificial Intelligence, vol. 114, no. 1-2, pp. 3-55, 1999.

[11] O. Wulf, K. O. Arras, H. I. Christensen, and B. Wagner, "2D mapping of cluttered indoor environments by means of 3D perception," in IEEE/RAS Int. Conf. on Robotics and Automation (ICRA), New Orleans, USA, 26 Apr. 2004.

[12] M. Walther, P. Steinhaus, and R. Dillmann, "A foveal 3D laser scanner integrating texture into range data," in Intelligent Autonomous Systems 9, 2006.

[13] J. Ryde and H. Hu, "Laser based simultaneous mutual localisation for multiple mobile robots," in Proc. of IEEE Int. Conf. on Mechatronics and Automation, Niagara Falls, Canada, Jul. 2005, pp. 404-409.

[14] — "Mutual localization and 3D mapping by cooperative mobile robots," in Proc. of Int. Conf. on Intelligent Autonomous Systems, The University of Tokyo, Tokyo, Japan, Mar. 2006.

[15] — - "Cooperative mutual 3D laser mapping and localization," in IEEE Int. Conf. on Robotics and Biomimetics, Kunming, China, Dec. 2006.

[16] R. Rocha, J. Dias, and A. Carvalho, "Cooperative multi-robot systems: a study of vision-based 3-D mapping using information theory," Robotics and Autonomous Systems, vol. 53, no. 3-4, pp. 282-311, 31 Dec. 2005. 